\title{
A synthesis of research findings on quality of services in the Indian Family Welfare Programme
}

Gillian H.C. Foo

Follow this and additional works at: https://knowledgecommons.popcouncil.org/departments_sbsr-rh

Part of the Demography, Population, and Ecology Commons, International Public Health Commons, and the Quality Improvement Commons

How does access to this work benefit you? Let us know!

\section{Recommended Citation}

Foo, Gillian H.C. 1996. "A synthesis of research findings on quality of services in the Indian Family Welfare Programme," Asia and Near East OR/TA Project Special Report. New Delhi: Population Council. 


\section{A SYNTHESIS OF RESEARCH FINDINGS ON QUALITY OF SERVICES IN THE INDIAN FAMILY WELFARE PROGRAMME}

Prepared by

Gillian H-C. Foo

PROCEEDINGS FROM THE NATIONAL WORKSHOP ON OPERATIONS RESEARCH FOR IMPROVING QUALITY OF SERVICES Bangalore, Karnataka 24-26 May, 1995

The Population Council, India 1996 


\section{CONTENTS}

I. INTRODUCTION 1

Objectives of the Workshop 2

II. USERS' PERSPECTIVES 3

A. Accessibility and availability of services 3

B. Availability of essential supplies 5

C. Method choice 6

D. Information provided to clients 6

$\begin{array}{ll}\text { E. Technical competence } & 7\end{array}$

F. Follow-up and mechanism for continuity of use 7

G. Client-provider information 8

H. Satisfaction with services 9

I. Summary 9

III. PROVIDERS' PERSPECTIVES 10

A. Accessibility and availability of services 10

B. Logistical support 12

C. Method choice 14

D. Comprehensiveness of information provided 15

E. Technical competence 15

F. Follow-up and mechanism for continuity of use 16

G. The effect of targets on the performance of providers 17

H. Summary 18

IV. QUALITY OF CARE IN STERILIZATION CAMPS 19

$\begin{array}{ll}\text { A. Standards of infrastructure } & 19\end{array}$

B. Technical competence 21

C. Interpersonal dimension of quality of care 25

D. Organization and management of camps 25

E. Summary 26

V. IMPACT OF QUALITY OF CARE ON CONTRACEPTIVE USE 27

A. The effects of quality of care on contraceptive adoption 27

B. The effects of quality of care on contraceptive continuation 28

VI. DISSEMINATION AND UTILIZATION 30

\section{APPENDICES}

I. List of Papers

II. List of Participants

III. Agenda 


\section{QUALITY OF SERVICES IN THE INDIAN FAMILY WELFARE PROGRAMME}

\section{INTRODUCTION}

In recent years, there has been growing recognition among researchers and policy makers that the quality of care provided by family planning programmes in developing countries is an important determinant of whether couples adopt contraception and continue as contraceptive users, or whether these programmes are under-utilized by the population they are designed to serve. Quality services are also considered to be basic to individual women's overall reproductive health needs.

Although quality of care issues have generated considerable research interest, and theoretical frameworks have been developed for defining quality of care (most notable is that by Bruce and Jain of the Population Council), the absence of systematic empirical evidence on the relationship between quality of care and contraceptive use is one reason why this dimension has not been accorded greater priority. This is true of the Indian government programme where policy makers and programme managers have been slow in introducing improvements in the quality of services. Another reason contributing to this absence of effort is that discussions on this issue remain at a very general level and concrete recommendations on programming improvements are elusive. Today, the Indian programme's achievements remain moderate, 40 years of effort notwithstanding. The programme is characterized by centrally established contraceptive targets--especially for sterilization--which are deterimental to the overall quality of services, limited method choice for couples who wish to either space or terminate childbearing, and major gaps in areas such as counselling, provider-client relations, and continuity of care.

A major initiative to identify and implement improvements in the quality of services within the Indian programme calls for a systematic analysis, organisation and presentation of the available data to the policy makers and programme managers concerned. Additional data, especially qualitative information, is also required on issues such as user's perception of quality of care, the relative importance of various components of quality of care (e.g. choice of methods, technical competence, interpersonal relations), as well as quality of care from the provider's perspective.

In response to these gaps in information on the quality of services, the Population Council, with support from the Ford Foundation and the United States Agency for International Development (USAID), commissioned a series of studies on the quality of services provided by the Indian government family planning programme, and organised a workshop where these research findings could be presented. This report synthesizes the findings of the 28 research papers presented at the workshop on "The Quality of Services in the Indian Family Welfare Programme" held in Bangalore from May 24 to 26, 1995. 


\section{Objectives of the Workshop}

The general objective of the workshop was "to synthesize available evidence on the standards of care provided by the Indian programme and the relationship between quality of care and effective family planning use." Furthermore, it was to suggest areas for further operations research, as well as possible policy and programme interventions. The specific objectives included in the following:

1. To present empirical data available on:

- Users' perceptions of "good quality of care";

- Providers' perceptions of the quality of services provided and the problems faced in providing "good quality of services"; and

- Linkages between quality of services provided and contraceptive prevalence as well as continuation of use of temporary methods.

2. To provide a forum for programme managers, from both central and state government, and researchers to discuss the quality of care provided in India and to identify possible areas for operations research and interventions to improve it.

3. To discuss possible procedures for institutionalising quality of care as an essential service component.

This report is divided into four sections: 1) Users' Perspectives, 2) Providers' Perspectives, 3) Quality of Care in Sterilization Camps, and 4) the Impact of Quality of Care on Contraceptive Use. 


\section{USERS' PERSPECTIVES}

A major objective of this workshop was to identify clients' perceptions of the quality of care provided by the Family Welfare Programme with respect to family planning services, both in terms of the various dimensions of care received and the nature of clients' interaction with service providers. The workshop research papers on this topic drew information from quantitative surveys as well as from qualitative studies based at both clinic and outreach settings in the following states: Maharashtra (Murthy), Karnataka, Tamil Nadu; West Bengal, Bihar (Roy and Verma), Madhya Pradesh (Barge), Kerala (Ramanathan) and Tamil Nadu (Ravindran). The data were gathered through diverse methodologies which included surveys, client-flow analysis, observations, interviews and focus group discussions. The following discussion is a synthesis of the findings of these papers, organized according to specific indicators of quality of care which are standard to these papers.

\section{A. Accessibility and availability of services}

The issues discussed in this section attempt to ascertain whether the family planning services offered are accessible to clients, if they are available, and if they meet clients' needs. At the outreach level, the issues considered are frequency of household visits by the Auxiliary Nurse Midwife (ANM) and the amount of time spent with a client. At the clinic level, the issues considered are clinic timings, availability of a physician, length of wait to see a physician, availability of medication, and users' source of contraceptive supplies.

\section{Outreach: frequency of household visits}

Within the government program, the norm is that at the village level, a multipurpose extension health worker should visit every household within his or her work area at least once in two months to provide family welfare services. These include disease surveillance, information on and services related to maternal and child health, as well as motivation for family planning and the provision of some contraceptive methods. In rural Maharashtra, Murthy found that ANMs had visited only 50 percent of the households they should have in the prior three months (and male workers even less). In Bihar, 53 percent of respondents reported a home visit within the same time period, and in West Bengal this figure was 60 percent. Home visits by health workers in Tamil Nadu and Karnataka were more frequent--with 92 percent and 81 percent of respondents reporting a recent visit. However, Barge's qualitative data from Madhya Pradesh support the observation that ANMs' visits to outreach areas are irregular. The community of one ANM's work area made it clear to the researchers that neither the ANM nor her male counterpart had visited their village in the past six months and thus had little credibility. Villagers preferred private clinics over government facilities.

\section{Utilization of government clinics}

Visited in last six/twelve months. In order to obtain an indication of the extent to which government health services are utilized, women respondents who were interviewed in the two surveys which provided data on client perceptions of government family planning services (Roy and Verma, and Murthy) were asked if they had used a government health facility within the past 
year. Although more than half of respondents (53 percent) reported having visited a Primary Health Centre/Sub-Centre (PHC/SC) in the past year, three-quarters ( 76 percent) had been to a private practitioner in the same time period. In their study of family welfare services in four Indian states, Roy and Verma found that while the majority of women had been to a government health facility in the past six months, a lower proportion of these same women in Karnataka, Bihar and West Bengal stated that they would go to a government facility in case of illness. Clients' perceptions of private sector health and family planning services as superior to those offered by the government program are clearly illustrated in Murthy's findings whereby respondents consistently rank specific dimensions of service quality higher in the private sector compared to the public. In addition, as Ravindran suggests, the shortage of women doctors in the public sector clinics may draw more women clients to private clinics where women doctors are more likely to be found. Nevertheless, government clinics continue to be utilized--substandard service notwithstanding--because their monetary costs to clients are minimal.

\section{Clinic: accessibility to clients}

The physical accessibility of a clinic is location-specific and further depends on a client's residence as well as on factors such as availability of transportation. At the same time, accessibility is also determined by clinic timings--whether these hours are convenient to clients and whether these stated working hours are actually observed by staff. In these studies, the majority of respondents stated that the timings of the government health facility which they use were convenient. However, whether the facility is actually open during the stated hours is a different matter. At half of the health facilities studied in Tamil Nadu, the timings were unpredictable. The reliability of opening hours appears to diminish as one moves to a lower-level facility (i.e. from PHC to SC) which tends to be poorer in infrastructure, equipment and supplies, staffed by a provider who is less likely to be resident there, and who is also less likely to be motivated since the services she can offer are limited given the inadequate resources. Where a health facility was only open in the mornings, women complained that the hours were inconvenient as they conflicted with household duties.

\section{Clinic: availability of medical personnel}

The presence of medical personnel, particularly of a doctor, is central to the availability of services. The findings of these studies show that doctors are not always present: two out of the four PHCs studied in Kerala had no doctor, and similarly, in Maharashtra, only half of the 14 physician posts at the PHCs studied were filled. As the study of services in four-states underscores there exists great variation by state. While in Bihar, only 30 percent of respondents reported that a doctor is always available in a government facility when needed, the corresponding figure for both Karnataka and Tamil Nadu is 64 percent. In Maharashtra, in contrast to the 64 percent of respondents who considered a doctor to be always available in a government clinic when needed, the percentage who viewed this as applying to a physician's availability in the private sector was 87 percent. The contrast between a physician's availability in the private as opposed to the public sector is accentuated by the fact that in some cases, physicians are not available at government facilities because they are carrying out their private practice during government hours. According to Ravindran, when doctors were not available for the duration of the clinic, "they were usually available for two to three hours a day, and carried out private practice in the afternoons." 


\section{Clinic: waiting time}

Waiting time to consult a physician varies considerably, but these studies have not isolated the factors that determine the length of time a client has to wait. For example, in Bihar, only 30 percent respondents reported that a physician was readily available at a government clinic, yet 50 percent reported a waiting time of under half an hour for consulting a government doctor. In Maharashtra the gap between the waiting time required for a government as opposed to a private doctor was not as large as expected. Exit interviews with women patients at government clinics in the four-state study found that with the exception of West Bengal, under 10 percent were subject to a wait of an hour or more to be seen by a doctor; in West Bengal, 35 percent reported a lengthy wait.

\section{B. Availability of essential supplies}

\section{Clinic: availability of medicines}

A physician or a health facility's perceived efficacy is governed by a successful response to a patient's health care requirements and this, in turn, depends upon the provision of the appropriate medication. There is extensive variation in the availability of medication at government clinics by state, with 52 percent of respondents in West Bengal stating that adequate medication is rarely available and conversely, 67 percent in Tamil Nadu reporting medication to be always available. Exit interviews with women clients at government health facilities confirm this considerable variation by state, with approximately half of the respondents in the West Bengal and Bihar surveys reporting that they had been given the required medication, whereas the corresponding figures for Karnataka and Tamil Nadu are over 80 percent. Nevertheless, in Tamil $\mathrm{Nadu}$, Ravindran found that there was an "acute shortage of drugs and essential supplies," so that while all levels of health facilities may dispense drugs, these were the common curative medicines for pains and fevers, colds, coughs and diarrhoea, and that for other medications patients were given a prescription which must be purchased from a pharmacy.

Women being admitted to government facilities for institutional deliveries had to bring the home birthing kits distributed by ANMs given the shortage of supplies, and had to purchase not only blood and saline but gauze and cotton wool. Although respondents consider medication to be more readily available in the private sector, Murthy suggests that clients do not resent the practice in government facilities of giving prescriptions for medicines not in stock, since private physicians may also give prescriptions for medicine to be bought elsewhere, rather than dispensing directly.

\section{Availability of contraceptives}

In terms of contraceptive supplies, although the majority of respondents in the study of four states expressed a preference for government sources, a higher proportion in Bihar and West Bengal--the states with the weaker infrastructure and poorer services--said they would use private sector sources. A very low proportion of respondents who were interviewed about 
outreach services reported distribution of contraceptive supplies by ANMs: in Karnataka and Tamil Nadu, the figures are four percent and two percent respectively, and 13 percent for Bihar and West Bengal.

The following discussion considers the issues of method choice and the technical competence of providers as perceived by the users.

\section{Method choice}

Key to quality family planning services is the issue of method choice which implies that the client is provided full information on all contraceptive methods: how they work, their advantages and disadvantages, associated side effects and treatment of side effects. All the studies discussed here found that clients received very little information from providers in the way of method choice. In Maharashtra, over 50 percent of potential acceptors who were interviewed about providers' outreach performance said that they were not told about spacing methods, and a similar proportion reported that they were not advised to use a method. However, Murthy found that those potential acceptors who claimed to have received information on methods did not report the expected undue emphasis by providers on sterilization, thus suggesting that ANMs would appear to be promoting method choice. In contrast, in the four-states study, method choice is clearly limited and at clinics, female sterilization is the most widely emphasized method, especially in Karnataka and Tamil Nadu.

Among respondents interviewed about outreach services, sterilization is reportedly also the most promoted method, except in Karnataka where ANMs promote both sterilization and spacing methods. Ravindran cites cases of involuntary acceptance of a method in Tamil Nadu--for example, IUD insertions and sterilization without consent, and making the availability of an abortion provisional upon a woman agreeing to be sterilized--but it is difficult to gauge how extensive these cases are.

\section{Information provided to clients}

These studies demonstrate that information given to clients by providers on family planning methods is inadequate, that side effects are not clearly delineated, and that clients are not counselled in how to deal with them. From exit interviews with women in Tamil Nadu and West Bengal who have gone to government clinics for family planning services, it is apparent that contraceptive users are not given comprehensive information on each method: under two-thirds of these women reported having received a description of the method and how it works. A notably smaller proportion (47 percent for Tamil Nadu and 40 percent for West Bengal ) had received information on side-effects, and even fewer (40 percent and 37 percent, respectively) had been told what to do if they were to experience side effects. Qualitative data from Kerala support these survey findings on the incomplete nature of information on contraceptive methods received by family planning users from providers. Providers tend to highlight a contraceptive's effectiveness and avoid raising other relevant issues which may be less positive, such as

contraindications. Roy and Verma found in the four-states, providers deliberately withhold information on side effects from their clients. In terms of who would help clients deal with side 
effects, under half the acceptors in the Tamil Nadu survey and 39 percent in West Bengal stated that a provider would counsel them concerning problems. While most of the respondents in Maharashtra who had experienced side effects had access to public sector medical care, less than half stated that they would go to a government facility for dealing with these, preferring instead a private doctor.

\section{E. Technical competence}

The issue of technical competence on the part of providers is discussed more fully in the section on providers. These studies could have addressed the issue of clients' perspectives on providers' competence more adequately. In the qualitative studies where the issue of provider competence is considered, Barge cites a case of an ANM who is frank with her outreach clients about her own absence of confidence in her ability to insert an IUD, and Ravindran reports poststerilization and post-MTP infections, and cases of sterilization failure which have "led to a general perception that laparoscopy was associated with high failure rates." In the Maharashtra survey, respondents were asked if they had been examined properly at government clinics, and whether they considered the treatment they had received to be effective. Respondents rated these aspects of providers' competence less favourably at government facilities than that at private clinics.

The respondents of the exit interviews in Roy and Verma's study were asked if they had received the required medicines, received other needed services, and received adequate attention. Respondents in Bihar and West Bengal reported a markedly lower level of quality on the three dimensions of care received from providers in comparison to respondents in Tamil Nadu and Karnataka. Respondents in the same study who had received outreach services were asked to rank the frequency with which their ANM paid attention to the client's family planning and family health needs. As is consistent with the other data from this study, respondents in Tamil Nadu and Karnataka reported a higher level of provider competence than did respondents in Bihar and West Bengal.

\section{F. Follow-up and mechanisms for continuity of use}

Related to the issue of contraceptive side effects is that of follow-up and continuity of services--and thereby continued contraceptive use. Absence of follow-up is an acknowledged failing in the government programme. In both Tamil Nadu and West Bengal, only half of the women who participated in the exit interviews reported that they had been given an appointment for a follow-up visit with the provider. Similarly, Ramanathan found that only seven out of the 22 cases observed at the PHC level in Kerala were given a fixed time to revisit the PHC. Char, in a study on contraceptive continuation (discussed more fully in Section V), reports that the majority of IUD acceptors in urban Bihar, Orissa and Gujarat who were interviewed stated that they had received no follow-up house visit from a health and family extension worker subsequent to their acceptance of an IUD. 


\section{G. Client-provider interaction}

The following discussion focuses on client-provider interaction, an important aspect of quality of care, and considers various dimensions of such interaction.

Amount of time spent by provider with client. The amount of time a provider spends with a client is an important component of the overall quality of services received. At the outreach level, ANMs can only achieve the norm of 50 household visits a day if they spend under five minutes at each house. In reality, Barge found in following six ANMs on their outreach visits that ANMs spent an average of six hours a day in outreach activities, and that most of their time was expended travelling from the PHC to the villages, and socializing around the village, so that they had under half an hour for actually contacting clients. Half of the respondents in the Maharashtra survey reported that the ANM had spent between five to ten minutes on the household visit and 91 percent of them considered this to be adequate or more than adequate. From these responses, Murthy concludes that five to fifteen minutes is the optimal amount of time for the duration of an ANM's visit. She notes that although clients justify the brevity of an ANM visit by pointing out that ANMs must cover many households and generally do not have strong expectations about the amount of time an ANM should spend with them, they nevertheless expect ANMs to visit according to schedule.

In contrast to the outreach level, the survey-based studies did not ascertain the amount of time the provider spends with a client at the clinic level. From the qualitative studies which report on the duration of client-provider interaction at the clinic level, it is clear that there is a great deal of variability.

Interpersonal dynamics. The survey-based studies nevertheless attempted to measure clients' perceptions of the interpersonal dynamics between provider and client. Respondents in the outreach areas surveyed in Roy and Verma's study were asked to rank the frequency with which they considered their ANM to be sincere; exit interview respondents were asked if the doctor at the PHC had been cordial. Respondents in rural Maharashtra were asked to assess the frequency with which they considered staff at government and at private clinics to be friendly, the providers/staff to be attentive, and their questions answered. The inter-state variations in the findings are marked, with providers' interpersonal behaviour in Bihar and West Bengal receiving distinctly lower ratings than in Karnataka and Tamil Nadu. Respondents in Maharashtra rated providers' interpersonal behaviour lower in government clinics compared to private clinics.

The qualitative data on interpersonal dynamics are mixed: direct observations of clientprovider interaction tend to be positive, with researchers generally reporting empathetic, patient and kindly care on the part of the provider (see Barge's and Ramanathan's papers)--a result perhaps of bias introduced by the methodology. In contrast, Ravindran's data obtained from interviewees' reports of their encounters with support staff and providers in health facilities are overwhelmingly negative, ranging from verbal and physical abuse dealt by staff and nurses, to demands for money from patients before rendering the most basic but essential services in any medical procedure (e.g. cutting the umbilical cord during delivery, performing an enema, being transported on a stretcher). 


\section{H. Satisfaction with services}

Overall, a client's reported satisfaction with services is an indication of the quality of care and services received. Considering that Karnataka and Tamil Nadu consistently received high scores from respondents on the individual dimensions of quality service, it is not surprising that 98 percent of respondents in both states expressed satisfaction with the services received. More surprising is that given the comparatively poor ratings by respondents in Bihar and West Bengal to the same elements of quality, approximately 80 percent of them reported satisfaction with the services received. Qualitative findings in Kerala also suggest low levels of dissatisfaction in the face of indifferent quality of services. These findings are plausible because a) clients are less welleducated compared to service providers, especially doctors, and therefore do not question their treatment; b) rural clients in particular have few options in terms of health and family planning services; c) government clinics are free and as clients have low expectations, they are easily satisfied with low quality of services. Murthy found that clients are not able to adequately discriminate between high and low quality care and that they need to be better informed educated on these issues. Most significant, perhaps, is the finding that a client's perception of quality is "formed from a pattern of experiences" with the services offered, rather than from contact with any one single element of care, so that improvements in quality of care should be implemented comprehensively.

\section{Summary}

This review of the studies on clients' perspectives on the quality of care has found that clients perceive the private sector as offering health and family planning services that are superior in quality to those offered by the government. In addition, clients' assessments of the individual dimensions which compositely define quality of care display considerable interstate variation, paralleling the standards of services extant in the states. All these studies found that clients are generally not offered method choice, and that the information they are given by providers on individual contraceptive methods is extremely limited, with the issues of contraindications and side effects seldom raised. In spite of such marked deficiencies in the quality of care, clients' expectations are sufficiently low that the majority express satisfaction with the services they receive.

\section{PROVIDERS' PERSPECTIVES}

The factors that determine the quality of care provided by health workers, and their own perspectives on what constitutes quality care, are the subject of several studies within this project. The information in this section is drawn from studies conducted by Visaria in Gujarat; Khan, Patel and Gupta in Uttar Pradesh; Verma and Roy in Tamil Nadu, Karnataka, West Bengal and Bihar; and Iyer and Jesani in Maharashtra. Although these studies include information from service providers such as Lady Health Visitors (LHV) and Medical Officers, they largely focus on the Auxiliary Nurse Midwife (ANM) who is the key worker at the grass-roots level. ANMs 
at Primary Health Centres and Sub-centres were interviewed. Various dimensions of quality of family planning services from the providers' perspective were examined in these studies. These include contraceptive choice, the comprehensiveness of information offered to clients, the technical competence involved in the actual provision of methods, and whether providers followup clients. In addition, infrastructural and logistical support as well as adherence to public sector standards of care which have a bearing on the performance of service providers are assessed. This summary of the findings is organized by those specific topics that affect the quality of the services provided.

\section{A. Accessibility and availability of services}

\section{Work Area}

According to the norm prescribed by the government, one ANM should cover a population of 5,000 in non-tribal areas, and 3,000 in tribal areas. In reality, this norm is seldom realized as village size rarely conforms to such prescriptions. An ANM may be assigned to a village with a population larger than 5,000 or to two or more smaller villages with a combined population that invariably exceeds the 5,000 norm and requires commuting to serve. In Uttar Pradesh, of the 54 ANMs interviewed, 50 percent of them covered between 6 to 15 villages while one-third covered 16 or more villages over a 5 to $6 \mathrm{~km}$ radius.

\section{Vacant posts}

Exacerbating the problem of effective coverage is the high proportion of sanctioned positions which remained unfilled. In each of the four states studied by Verma and Roy, less than 50 percent of the PHCs were staffed according to the prescribed norm.

\section{Workers' Residence}

Central to the effective delivery of services and the ANM's accessibility to rural people is that ANMs should reside in their assigned village, ideally at the Sub-centre which should include living quarters as well as a building adequate for outpatient clinic facilities. However, the provision of living quarters does not necessarily occur. Iyer and Jesani found that in Maharashtra, of the ANMs in the four districts surveyed in their study, 83 percent of those posted at Subcentres had not been provided with living quarters. In practice, non-residence of ANMs appears to be a common problem. In Uttar Pradesh, under 50 percent of the ANMs interviewed were resident in their Sub-centre village. In Gujarat, residence varied by district. Factors cited by ANMs as determining residence are the condition of the building, its location and security, as well as available educational facilities for their children. ANMs stated a preference for living in bigger villages or nearby towns. The distance between their Sub-centre and their residence for nonresident ANMs in the Uttar Pradesh study ranged from 5 to $25 \mathrm{kms}$. Irrespective of the reasons, non-residence of health workers in their assigned work area results in extensive commuting which erodes the time available for actual service delivery, their frequent absence as well as contributing to their being less available during emergencies. 


\section{Barriers to covering work areas effectively}

In addition to vacant posts and non-residence, barriers to ANMs covering their work areas effectively center around the issues of transportation, security, caste and religion. Generally, ANMs travel to outreach villages by foot although for more distant villages they may arrange for vehicular transport such as bikes and scooters, with male escorts. Khan et al observe that the lack of transport and of a travel allowance result in workers only going to the more remote villages in the winter months during the annual drives for sterilization cases. Physical security related to mobility is another major concern of ANMs cited in these studies. Data for Maharashtra and Uttar Pradesh document the sexual harassment to which female health workers are subject, ranging from verbal teasing to rape. Fear of theft, robbery and sexual violence were mentioned by both ANMs and medical officers in Uttar Pradesh, and ANMs reported that there were certain villages they would not visit without a male escort. Caste and religion may also adversely affect a provider's efficacy. Health workers in Uttar Pradesh stated that it was difficult for them to work in certain areas where a particular caste or religion which was opposed to family planning was dominant. According to Visaria, an ANM's age and marital status may also affect her acceptance by the community, with younger and unmarried women having greater difficulty gaining acceptance within the community.

\section{Management of Time}

The study on Uttar Pradesh assessed ANMs' use of their actual work time. It was found that unproductive work occupies a large proportion of ANMs' time. For example, on the weekly vaccine collection day, ANMs go to the larger health center to collect their vaccine supplies and do no other work on that day. Similarly, on sterilization camp days, all ANMs are expected to assist at these camps whether or not a client of theirs is being sterilized, and irrespective of the case load which doctors say they are unable to anticipate. The researchers report that on one camp day there were 24 ANMs to a sole sterilization patient. Although ANMs are expected to perform two days of outreach work in a week, these days are not fixed and are thus subject to the exigencies of other activities such as the collection of salary or the monthly meetings of all health workers. When epidemics break out seasonally, all routine work is stopped and all effort concentrated on treating the epidemics. Invariably, because outreach work receives lower priority, less of it gets done than should.

\section{The ANM's work portfolio: Allocation of work priorities}

The ANM's work portfolio includes not only family planning but also maternal and child health. Thus the ANM's responsibilities involve immunizations and antenatal, natal and postnatal care in addition to motivating women to use temporary contraceptive methods or to become sterilized. Although the papers discussed here focus on the quality of family planning services primarily delivered by ANMs, Visaria's and Verma and Roy's studies also assess ANMs' performance where maternal and child health services are concerned. Visaria reports that while ANMs in Gujarat view MCH care as their priority, their supervisors consider family planning services to be of greater importance. 
In terms of ANMs' level of knowledge regarding MCH issues, Verma and Roy found this to be unsatisfactory in Bihar, Karnataka and West Bengal and satisfactory only in Tamil Nadu. With the exception of workers in Bihar, the majority of workers in the other three states were generally knowledgeable about child immunization schedules. In contrast, workers were unclear as to when the fertile period occurs in the menstrual cycle and were also ignorant of breastfeeding practices. Visaria observes that while ANMs appear to be well-versed in antenatal, natal and postnatal care, and are knowledgeable about complications and when to refer, it is uncertain whether this knowledge is actually implemented.

In practice, antenatal care clearly receives low priority. For example, under 50 percent of client respondents in all four districts reported receiving some form of antenatal care (tetanus toxoid immunization and iron and folic acid tablets) during their last pregnancy. The role of ANMs in terms of deliveries was also small. They are often reluctant to participate in deliveries because of their concern with their physical safety at night as well as the ensuing complications of participating in high-risk deliveries. In fact, client respondents said that they did not know that the ANM may be contacted for deliveries; they tend to use the local dai, the government hospital or a private practitioner instead. None of these studies looked specifically at the issue of postnatal care.

\section{B. Logistical Support}

Assessments of logistical support in these studies consider issues of space, condition of buildings, availability of electricity, water and toilet facilities, as well as the availability of essential equipment, sterilization facilities, medicines, vaccines, contraceptives and other supplies.

\section{Space and buildings}

The three studies which evaluated the conditions of the health facilities where the ANMs were based found these to be unsatisfactory. In Uttar Pradesh, very few of the clinic buildings are government-owned, with most being rented or donated. The majority of Sub-centres were one-room structures, without toilet facilities, electricity, or a generator. Drinking water was usually available, however. In Bihar, Karnataka, Tamil Nadu and West Bengal, waiting space with seating, an examination room and a consultation room with privacy, toilet facilities and drinking water were inadequate. Visaria notes that the maintenance of buildings is very poor. She further states that some of the ANMs in Gujarat function from their own residence, thus raising issues of space and privacy. Khan et al observe that not having a proper space from which to run a clinic provides the ANMs with an excuse for not opening the clinics or for not conducting physical examinations.

\section{Equipment and commodities}

These appear to be unevenly supplied. In the four states evaluated by Verma and Roy, an examination table, blood pressure instrument, vaccine-carrying box and scale were generally available. In contrast, in Uttar Pradesh, many Sub-centres did not have an examination table, weighing scale or reagents for performing urine and blood tests. While 70 percent of the ANMs had a blood pressure gauge, only 13 percent had a functioning stethoscope. Visaria observes that 
in Gujarat, while basic equipment may be provided, they commonly require a small maintenance budget and supplies such as chemicals and kerosene to be operable.

The importance of a maintenance budget and supplies is obvious where ensuring aseptic/sterile conditions for equipment at clinics is concerned. While 11 percent of the 54 Subcentres in Uttar Pradesh had neither sterilization equipment nor kerosene, a notable 56 percent had the equipment but not the kerosene, and only 33 percent had both. Physicians interviewed in this study stated that the monthly 30 rupees sanctioned for the purchase of kerosene never materializes. Antiseptic solutions were available in only nine percent of these Sub-centres. Onethird also had no disposable gloves so that ANMs performed IUD insertions and pelvic exams without gloves. The ANMs interviewed in Uttar Pradesh reported that they had to use their own money to purchase gauze and cotton wool as these were not supplied by the government as they should be.

Apart from iron and calcium tablets, all the studies noted a shortage of basic curative medicines and drugs for treating common ailments. ANMs complained that their patients lost confidence in them when they could not supply essential medicines and went elsewhere for medical attention. Where the supply of vaccines is concerned, in Uttar Pradesh one-third of the ANMs interviewed complained that at least one vaccine--and frequently more--was out of stock. This unreliable supply of vaccines poses problems with respect to following immunization schedules and achieving immunization coverage. Similarly, the supply of contraceptives was inadequate. Khan et al report that the modern reversible methods provided within the government programme were out of stock at both the Sub-centre and PHC levels in Uttar Pradesh. Five out of the ten PHCs evaluated had no IUDs in stock, and three out of ten had no oral contraceptives or condoms. Of the 54 Sub-centres sampled, one third of their ANMs had no IUDs or IUD insertion kits, so that kits were shared between ANMs.

\section{Support from other health providers}

In the field, the ANM works with the Male Multi Purpose Worker (MPW/M) and the local dai. Within the hierarchy of health providers, her immediate supervisor is the Lady Health Visitor (LHV). In Uttar Pradesh, the ANMs complained that in contrast to earlier times when they and the male workers did outreach work as a team, they no longer receive much support or cooperation from their male colleagues and are left to conduct outreach work on their own. They also complained that their male counterparts tend to be very authoritative and superior towards them while doing very little of the work. A physician observed, however, that male workers now have a far greater population to cover than ANMs and are thus not in a position to be helpful.

Where local dais are concerned, Visaria notes that in Gujarat, ANMs reported that the dais were generally supportive of them and helped with identifying pregnant women for antenatal care. According to data from the states studied by Verma and Roy, ANMs' contact with their supervisors is infrequent. When it does occur, supervisors mainly check stocks and supplies. One-quarter of the ANMs reported receiving guidance from their supervisors, while only onetenth said that their supervisors actually helped them with client problems. 
In view of the absence of logistical support--from transport to medical equipment and supplies, ANMs find it difficult to perform their job and to provide basic services, let alone quality services.

\section{Method choice}

The policy of the Family Welfare Programme is that clients should be able to voluntarily choose a contraceptive method (either terminal or temporary) from the full range of methods available, and that they should be provided full information on these methods. In practice, this does not occur. The studies cited here which have examined the issue of contraceptive choice were consistent in their finding that clients are rarely offered the range of contraceptives methods available in the programme. Verma and Roy report that 93 percent of ANMs interviewed in Karnataka stated that it is the providers who suggest the method to the client, and in the other three states, approximately 75 percent of the ANMs were of this opinion. Khan et al concluded from the focus group discussions conducted with ANMs in Uttar Pradesh that providers push a specific method according to the parity of the woman and the gender composition of her children and, as such, believe that they are promoting method-mix.

Observations of client-provider interactions corroborate this conclusion that clients are not offered the range of methods from which they can make an informed choice. Visaria found in Gujarat that only five percent of those sterilized women who had been motivated by both health and non-health functionaries had been offered a choice of method other than sterilization. Similarly, 88 percent of IUD acceptors said they had received no information on any other method. In the four-state study, with the exception of West Bengal (where ANMs promote spacing methods over female sterilization), ANMs are likely to promote female sterilization and the IUD, and to a lesser extent oral contraceptives. This study as well as that of Khan et al found that male sterilization is rarely offered as an option. At the same time, the shortages and erratic supply of contraceptives (i.e., IUDs, IUD insertion kits, and oral contraceptives) make it unrealistic for providers to offer clients a choice of methods. In Uttar Pradesh, all levels of the health workers interviewed referred to the constant logistical problems as a major reason why contraceptive choice for clients is not always feasible.

\section{Comprehensiveness of information provided}

Even though ANMs do not offer their clients a choice of methods, another issue is whether they provide their clients with complete information on the method or methods that they are promoting. Khan et al observed that although ANMs in Uttar Pradesh were very knowledgeable when interviewed about the different methods, how they work, and their side effects, they provided very little of this information to their clients. Clients were not told about the side effects of the contraceptives and were only asked to return if they have "any problem". They did not even explain to clients how the condom should be used, simply assuming that they would know.

Verma and Roy asked providers what information and advice they would give to a new acceptor of oral contraceptives. In all four states, a high proportion of ANMs do not ascertain 
their clients' reproductive goals, are reluctant to discuss possible side effects of the method, do not give clear directions as to how to use the pill, and do not advise clients to make a follow-up visit within a month.

\section{E. Technical competence}

There are specific protocols to be followed for each contraceptive method which screen clients to ensure that the method is not contraindicated.

IUD. Visaria reports that of the IUD users interviewed in her study, approximately half had received a physical check-up before insertion, over 40 percent stated that the provider had washed his/her hands or worn gloves, and two-thirds had received a follow-up visit by a health worker at home. In the Uttar Pradesh study, the majority of ANMs said that they would take a client's medical history and perform a pelvic exam prior to an IUD insertion, whereas only a third mentioned blood and urine tests and screening for a reproductive tract infection (RTI). Discussions with these ANMs reveal that very few actually perform pelvic exams on potential IUD acceptors, and that of those who do, many do not have gloves and so do the exam without gloves.

An important finding of this study is that ANMs may perform an insertion even though they find that the client is contraindicated for the IUD (for example, if they detect a reproductive tract infection prior to insertion) since they do not want to lose a motivated acceptor. Other method options are not offered. In some cases, if a client is rejected for an IUD, the ANM neither explains the reason to her nor refers her to a larger health facility for treatment. In terms of the actual insertion, ANMs admitted that as they had performed only three to four insertions during their training, they had little confidence in their own ability where insertions were concerned. This factor, together with the lack of facilities at Sub-centres, force potential acceptors to go to the larger health centres for IUD insertions.

Oral Contraceptives. Verma and Roy assessed technical competence of providers on the basis of their knowledge of the protocols to be followed in supplying oral contraceptive pills to firsttime acceptors as well as to women wanting a resupply of pills. Although there was inter-state variation in these findings, it was clear that providers tend to ignore verifying the potential acceptor's suitability for the method against the standard checklist. For example, less than half of the ANMs interviewed stated that they would measure blood pressure. Khan et al found that ANMs in Uttar Pradesh were well-versed in the procedures they should follow in screening a potential oral contraceptive acceptor, but they did not ascertain if these were followed in practice.

Sterilization. The quality of sterilization services is discussed in greater detail in Section IV which deals specifically with this issue. The following are findings on the provision of sterilization services from the providers' perspective which are common to the studies on this topic.

- Male sterilization is now rarely offered by providers as a terminal method. Providers cite the normative objections which the client population hold (e.g. causes weakness in men, leads to impotence) as well as the doctors' lack of skill in performing vasectomy surgery, as reasons for not offering this method. 
- Because of the method-specific targets imposed on health workers by the government, potential sterilization acceptors have become prized clients who are assiduously courted by the ANMs. However, an ANM accompanies her client to the place where the surgery will be performed as much out of a sense of responsibility to the woman as from a fear that another motivator (such as a functionary of the Revenue Department) will take over the client and claim credit for her acceptance of sterilization.

- ANMs frequently target women with unwanted pregnancies to be sterilization acceptors and tie the provision of a Medical Termination of Pregnancy (MTP) to an agreement to be sterilized. Khan et al found this to be common among the ANMs interviewed in Uttar Pradesh.

- In order to meet targets, the sterilization procedure is performed even though a woman may be contraindicated for this at the time of the surgery--by a low haemoglobin count, for example.

- Although basic care is provided at the sterilization camp, and clients receive the requisite health check-up, tetanus toxoid injection and general anaesthesia, the high proportion of women complaining about pain during the procedure suggests that the anaesthesia may have worn off or may be inadequate in dosage.

\section{F. Follow-up and mechanisms for continuity of use}

A finding common to these studies is that ANMs do not maintain their registers adequately to follow-up family planning acceptors. ANMs did not have a clear idea as to how many of their clients were still using a specific method, how many had discontinued and for what reasons. Researchers observed that ANMs did not appear to comprehend the importance of maintaining accurate and updated registers so as to facilitate follow-up but noted that the inadequate maintenance of registers is likely related to the falsification of records and of method-specific acceptors. These studies report that ANMs admit that it is common practice to inflate workers' performance where recruiting acceptors of reversible methods is concerned. When workers report an inflated number of IUD acceptors, they destroy or throw away the IUDs which they erroneously claim to have inserted.

Proper follow-up is an essential component of quality care, yet a consistent finding in these studies is that although ANMs consider it important to follow-up clients who have been sterilized, few follow-up IUD acceptors and none considers it necessary to visit acceptors of oral contraceptives. Workers visit sterilization clients to remove stitches or to refer them for further follow-up at the PHCs with a physician. Workers are also conscientious about following-up sterilization cases since it is a community expectation that the health worker visits a client after surgery. Sterilization acceptors in Gujarat complained that ANMs were not sympathetic to their complaints. ANMs, on their part, felt these complaints were related less to the surgery and more to the generally poor health condition of these women. Follow-up of IUD acceptors is not a common practice among ANMs; few consider it necessary and instead instruct IUD acceptors to contact them if they encounter any problems. ANMs rarely follow-up acceptors of oral 
contraceptives, assuming that users should not have problems and that if they do, will either discontinue the method or will contact a health worker.

\section{G. The effect of targets on the performance of providers}

These studies clearly document the overwhelmingly negative impact which numerical method-specific family planning targets have on the performance of health providers, and the ways in which they hamper a health worker's ability to offer quality care. Iyer and Jesani propose that by making a provider's professional behaviour entirely oriented to this system of targets, a dichotomy between curative care and family planning services is invariably established. Basically, non-family planning areas of health services which the client population require are neglected since a health worker is publicly penalized for not adequately meeting family planning targets. As Visaria observes, the existence of family planning targets mitigates against the integration of family planning with maternal and child health.

Providers admitted to researchers that they were under extensive pressure to meet the targets imposed on them. This pressure is reflected in their resorting to professional behaviour which is essentially unethical and which makes providing quality services unfeasible, including: 1) falsification of records by inflating the number of acceptors, especially those of reversible methods; 2) proceeding with a surgical sterilization or an IUD insertion when a potential acceptor's physical examination shows that the procedure is contra-indicated; 3) in some cases, refusing to assist or treat an acceptor suffering from side effects and complications who was motivated by a functionary from a different government department; 4) not providing a client with full information on the range of contraceptive choices available or on the side effects of the method proposed; or 5) making the availability of a medical termination of pregnancy (MTP) to a client with an unwanted pregnancy contingent upon her agreeing to undergo sterilization.

\section{H. Summary}

As a prelude to examining quality of services according to specific issues, Khan et al asked the ANMs in their Uttar Pradesh study for their perceptions of the services they were providing. These ANMs were unable to define quality, to identify gaps in their services or to propose improvements: they were generally satisfied with their work. The majority of them were unable to specify any particular areas of refresher training from which they would benefit. Medical Officers felt that the inadequacy of infrastructural facilities and logistical supplies together with the late payment of salary and the lack of a travel allowance, mitigate against the provision of quality services. The discussion in this section has highlighted the negative effect of method specific family planning targets on the quality of services offered by family planning providers as well as the need to arrive at commonly agreed-upon standards of care which guide providers' performance. 


\section{QUALITY OF CARE IN STERILIZATION CAMPS}

In India, male and female sterilization are the most common methods of contraception, and account for three out of every four contraceptive users. Although male sterilization-vasectomy--greatly outnumbered female sterilization in the early years of the government program, constituting 75 percent of all sterilizations in the mid 1970s, there has been a precipitous decline since then, and male sterilization accounted for only five percent of sterilization cases at the national level in 1993. Female sterilization is now the predominant method of sterilization, and within the public sector the procedure is frequently offered in "camps" so as to maximize the number of women on whom the procedure may be performed. This section discusses the findings of four studies which examined the quality of care of sterilization services provided at these MOHFW-convened camps, focusing on the standard of the infrastructure and facilities available, as well as the technical competence of the staff. The papers on these four studies are by 1) Townsend, Khan and Gupta on Uttar Pradesh; 2) Mavalankar and Sharma on Gujarat; 3) Lakshmi and Barge on Madhya Pradesh; and 4) Parveen on Rural Bihar.

According to Townsend et al, a camp is defined as “....any grouping of patients for a specific service, independent of the site, or quality. Operationally, even when services are provided at a health facility, such as a PHC or post-partum center, the event is labelled a camp when either personnel (e.g. surgeons, anesthesiologist) or materials (e.g. medicines, equipment) are supplied externally." Sterilization camps may be convened at the various levels of health centers--Sub-Centre, Primary Health Centre, Community Health Centre, Post-partum Centre, District Hospital--as well as at public institutions such as schools where a building is available. Acceptors are recruited by health workers and development sector staff (of the Revenue Department), but the services at these camps are provided by the staff of the MOHFW and they are responsible for the quality irrespective of the site where the camp is held.

\section{A. Standards of Infrastructure}

There are specific standards recommended by the government for the facilities where sterilization procedures are provided. The inter-state studies have found that the majority of facilities rarely meet these guidelines although there is considerable variability according to the level of facility (e.g. Sub-centre versus Post-partum Centre). The findings with respect to standards of infrastructure can be summarized as follows:

\section{Buildings and Utilities}

Government guidelines stipulate that the operating theatre (OT) should be at least 10 feet by 10 feet with an entrance and exit, that it should be well-lit with electricity as well as a working generator, and that there should be clean, running water and toilets. The following conditions are not required by the government but are necessary to ensure that the facilities are conducive to the provision of quality services. There should be a waiting area with seating, a room with visual and audio privacy where a patient can be screened and prepared for surgery (shaving and administered an enema) by the providers, and a recovery room for patients after surgery as the effects of the anaesthesia wear off. Since most patients are accompanied for sterilization by one 
or several relatives, space should also be made available for them as patients are usually at the facility for the duration of the entire day, commonly with infants and young children. In addition, these relatives should ideally have access to food and drink and toilets.

Standards of infrastructure, equipment and logistical support vary according to the level of health facility where the camp is convened so that Post-partum Centres and Community Health Centres tend to have more adequate facilities, equipment and trained staff than do PHCs and Subcentres. In the latter two centers, the operating theatre is usually a converted room with little audio and visual privacy. Outreach camps held at non-health institutions appear to have the least adequate facilities: they are dark and offer little privacy; they are frequently without clean running water in the room serving as the makeshift operating theatre, and without electricity. In such cases, water has to be carried in and lighting obtained from battery operated flashlights or from power derived from a vehicle battery. In these camps and in those held at the smaller health centers where the electricity supply is irregular, researchers observed that there were occasions when surgery in progress, with the patient opened up on the table and the laparoscope inserted in her, was interrupted for several minutes while waiting for the power situation to be resolved.

Irrespective of type of setting, a finding common to all the studies on sterilization camps is that toilet facilities are either non-existent (outreach camps) or, where they are available, are not functioning and/or filthy. Toilet facilities are particularly important because patients are administered an enema prior to the surgery. Inadequacy of space was also another finding common to all these studies. Waiting facilities were either very limited, or non-existent, and patients were observed to be sitting on the floor even after being administered their pre-operation medication. The recovery area for post-operation patients is equally inadequate: there is usually no privacy and patients are carried out and placed on mattresses (without sheets) or mats on the ground. If the case load is heavy, patients are tightly packed in close proximity to one another. The needs of relatives accompanying sterilization cases are never taken into account. There is no waiting space for them and ANMs report that they have to bring tea and biscuits for their patients' relatives which they pay for themselves.

\section{Equipment and Logistical Support}

There is a specific set of equipment required for surgery and for the administration of anaesthesia in addition to the instruments needed for the sterilization procedure. An autoclave and kerosene are required for maintaining sterile/aseptic instruments, linens and dressings. Sterile gloves which may either be disposed of or sterilized between each patient and clean gowns for patients should also be available. Stretchers are necessary for moving patients from one room to another. A vehicle should be available for transporting patients home after they have recovered from surgery as well as for bringing staff to the camp site. Staff should be trained in the sterilization procedure, and assistant staff should also be trained.

Again, the larger health centers are better equipped. Physicians commonly bring the required surgical instruments with them to camps held at the smaller health centers or non-health institutions. At the majority of camps, standard theatre equipment is either non-existent or in extremely poor condition, and use of improvised equipment is reported in more than one study (e.g. makeshift operating table). Some surgical instruments are reportedly barely functional and 
some literally held together with tape. Operating theatre apparel (gowns, masks, slippers) are in short supply so that these are not changed between patients. The adequacy in supply of gloves varies by facility, but it is clear from these studies that providers are not scrupulous about changing gloves between patients. Gowns are rarely provided to patients: they go in for surgery with their petticoats pulled up around them in lieu of gowns and are thus exposed to male staff. Stretchers/trolleys are not available in many places so that patients are usually carried into and out of the operating theatre by a male attendant.

\section{B. Technical competence}

The quality of health services is affected as much by the providers' willingness or ability to comply with institutionally defined standards of care as by the infrastructure. The discussion in this section focuses on the technical competence of staff providing surgical sterilization services at camps, and is organized according to pre-operation, surgical and post-operation care. It begins with a summary on the findings concerning cleanliness and hygiene and the maintenance of aseptic conditions at sterilization camps, issues where the intersection of infrastructure and professional behaviour are apparent.

\section{Cleanliness and hygiene of the facilities}

These vary by location. Descriptions range from dark and unaired operating theatres with bloodied swabs from several days back unswept on the floor and unchanged rubber sheets on the operating table (rural Bihar) to sites cleaned daily with phenyl and cleaned operating tables (Madhya Pradesh).

\section{The maintenance of aseptic conditions}

All the studies found that throughout the entire procedure of female surgical sterilization, the maintenance of aseptic conditions is extremely poor. Again this tends to vary according to the level of the facility, with the worst observation of aseptic precautions found at the outreach camps. The importance of maintaining sterile conditions where surgical procedures are involved does not appear to be recognized by providers of sterilization services. At the same time, this is exacerbated by the inadequacy of logistical support and supplies, the often heavy case load at these camps, and the time pressure providers tend to be placed under in these circumstances. The specific failures noted in observing aseptic precautions are as follow:

- With the administration of pre-operation medication, clean and adequately autoclaved needles and syringes are not used. They are commonly washed, in some cases in hot water, and may also be boiled for a few minutes, but for insufficient time to become sterile. The same needle and syringe are used to administer the different pre-operation drugs. Even where needles do not appear to be in short supply, camp organizers and nurses do not appear to take the trouble to prepare a sufficient number of autoclaved needles for the caseload.

- The same enema instruments are used for each patient without cleaning or washing.

- Similarly, the same razor blade is used for shaving more than one patient, without cleaning or disinfecting. 
- In the operating theatre, general aseptic precautions like changing gloves, gowns and caps after each patient are not observed.

- Health providers like the surgeons do not scrub prior to each operation. They also do not change gloves between patients; it is common practice for them to wash their gloves in hot water and to dip them in Cidex before moving on to the next operating table.

- Operating table sheets, although they may be freshly laundered and sterile at the start of the camp, are left on the table the entire day and not changed between patients.

- Sterilization of surgical instruments is highly inadequate. One set of researchers observed that instruments, including the laparoscope, are washed in hot water, dipped in Cidex germicidal solution for a minute or less (complete sterilization requires soaking for 10 hours and high-level disinfecting for 20 minutes), rinsed in water again, and then dried with a sterile cloth before use. Health centers may commonly start the day with autoclaved instruments, but rather than being properly sterilized again for the next patient, these used instruments are merely rinsed in hot water and dipped in Cidex for reuse on another patient.

- Sutures and dressings for the incision are also not sterile.

\section{Staffing}

The surgeons performing the sterilization usually commute to the camps at the smaller and outreach facilities and may be contracted from the private sector. Sterilization camps are organized by administrative sub-districts called talukas or blocks, are held once a week, and their staffing and management are shared on a rotating basis by the staff of the PHCs involved. Because it is a requirement that all ANMs from the block participate at the camp whether or not a client of theirs is being sterilized, the number of staff at a camp is considerable.

\section{Quality of Care Related to Surgical Sterilization Procedure}

The following section reviews the findings on the quality of care provided at sterilization camps related to the surgical procedure.

\section{Pre-operation Care}

Waiting time. The surgeons invariably arrive at the camps late so that patients are subject to a lengthy wait between the time they arrive at the camp and the surgery--one researcher reported the average waiting time to be 4 to 5 hours. This substantial delay poses a problem as patients have had to fast from the night before and are hungry.

Registration. This formality is properly completed as the incentive payment to sterilization acceptors requires the patient to be registered. 
Screening. Prior to surgery, the screening of patients by a systematic physical examination is essential in order to rule out high-risk cases. This involves the measurement of blood pressure, and urine and haemoglobin tests, as well as a general physical exam that includes a pelvic exam. The findings on whether screening was actually performed vary from none (Bihar), to cursory without a pelvic exam (Gujarat), to performed at all sites which the researchers observed (Madhya Pradesh and Uttar Pradesh). In Madhya Pradesh, clients were screened by a medical officer following registration, and there were those who were subsequently rejected for sterilization surgery. Rejected clients averaged two to three per camp. In Uttar Pradesh, it was observed that lab results were not always reported correctly, recorded haemoglobin levels were constant across all cases. This may have been done in order to retain women for the procedure who were actually anaemic and thus contraindicated for sterilization, so as not to lose a motivated client or another case toward meeting the targets.

Pre-operation preparation. This involves the administration of various medication (tetanus toxoid, anti-palpitation and anti-depressant drugs, antibiotics and anaesthesia) through a series of injections, an enema, and shaving the client. As discussed previously, a finding common to all the studies is that sterile syringes and needles, enema instruments and razors are not used for these procedures and are shared between patients without proper cleaning and sterilizing. Another common finding is that the stipulated time interval between the administration of the medication and the surgery is not observed, the full effects of the drugs are not felt, and the patient is caused much discomfort. In Bihar, it was observed that at outreach camps especially, there was occasionally an insufficient supply of anaesthesia for the case load so that the last few patients were administered a lower and inadequate dosage, and in one reported case, one ether bottle was shared between two patients simultaneously. While in Madhya Pradesh the staff appeared to be well-trained in pre-operative procedures, it was noted in Gujarat that they were not and had difficulty performing basic medical tasks such as opening the antibiotic valves and assembling the syringes and needles.

Pre-operation counselling. This seems to be non-existent at most camps. Patients were not informed of the procedural steps for the sterilization and did not know what to expect.

\section{Surgery}

At sterilization camps, the operating theatre commonly resembles an assembly line with two patients laid out on separate tables at the same time so that the surgeon can turn immediately from one to another as soon as he has completed the procedure. With a laparoscopy, the time taken from making the incision to the completed ligation is only 2 to 3 minutes, and the medical officer may suture and dress the incision so the surgeon can move on to the next patient. An average of 10 to 15 operations an hour can be performed with this system.

- It has been noted above that all these studies found that there is no proper observation of aseptic precautions in the operating theatre in sterilization camps. Operating theatre apparel for staff and sheets on the operating tables are not changed between patients, and surgical instruments, including the laparoscope, are not adequately cleaned and sterilized between patients.

- Operating theatre attendants are not well trained as they tend to be peons in the PHCs. ANMs who assist in the surgery are also reportedly not skilled as assistants since they have 
had no training in this area, are ignorant of the ideal procedures and have had to learn on the job. One study reports that an attendant laid out all the surgical equipment on an unconscious patient's leg prior to the start of the operation. In the same study, a case is also reported where the surgeon was unable to locate a patient's tube and it had to be found by the assistant.

- In the section on pre-operation procedures, mention has been made of the inadequate time interval between administration of anaesthesia and starting surgery, and the rationing of anaesthesia when the supply is insufficient so that patients receive less than the recommended dosage. Administration of one type of drug in lieu of another also reportedly occurs. At one facility, researchers observed that at the end of the surgery, after the incision had been sewn up, patients were given a sedative rather than the recommended painkiller which should be administered 15 minutes prior to surgery, with the result that patients were in acute pain.

\section{Post-operative care}

Following the operation, the patient is transferred to the recovery room and placed on beds, mattresses or rugs on the ground. Relatives then look after the patient. As stretchers are non-existent at most facilities, inert and unconscious patients are carried out in the arms of a male attendant to the recovery area. Some researchers state that this compromises standards of modesty since patients are not properly dressed (it was also observed that in some places, the male attendant tied the patient's petticoat). At many facilities where camps are held, the recovery area is not private. Medically, there is no monitoring of patients after surgery to ensure that they are not going into shock, and the surgeons leave as soon as the last operation has been performed, with only a cursory checking of the patients if at all.

Although it is recommended that patients only be discharged 4 to 5 hours after surgery, all the studies noted that they were discharged much sooner, in some places even before they were fully conscious. This premature discharge may be related to the volume of cases and the need for space. Before discharge, patients are given a packet of medication containing analgesics and vitamins and/or iron supplements, and in some places antibiotics. They are given minimal verbal instructions about how to take care of themselves (e.g. not to get the wound wet) but they do not receive written advice. Patients and their family attendants are then transported to their homes in a government-provided vehicle. In the one study which investigated the payment of the sterilization incentive, it was found that 15 rupees was deducted from the 150 rupees incentive fee to meet the cost of fuel involved in acceptors' transportation.

\section{Follow-up}

The ANM visits each patient at home following the surgery, often 2, 4 and 7 days after, to change the dressings, administer antibiotics if required, and after 7 days to remove the stitches. These studies have found that ANMs are conscientious about following-up sterilization acceptors and that their visits are regular. However, one study noted that ANMs are given no set protocol for these follow-up visits and thus have no guidelines with which to ascertain problems. 


\section{Interpersonal Dimension of Quality of Care}

The findings of these studies demonstrate that empathy with clients is entirely absent from sterilization camps and that no consideration is given to the dimension of inter-personal relations. Patients are treated with neither respect nor sympathy and are not provided with the most basic information which could make this a less anxiety-provoking experience. In one camp, two patients were made to squat on the floor in the operating theatre while waiting for the surgeon to complete surgery on the preceding two cases.

Observations in these studies indicate that nurses and doctors are intolerant of complaints from sterilization patients and respond to them impatiently and harshly. Medically, very little is done to ensure the physical comfort and safety of patients. Aseptic precautions are not observed, thereby exposing patients to infections and more serious morbidity through contaminated instruments. Medication is improperly administered so that patients are subject to acute pain during and after surgery. The modesty of women patients is not respected with the lack of spatial privacy at these camps, the inability to provide surgical gowns, and, it has been observed by Mavalankar and Sharma, by the gender division of labour among lower-level operating theatre staff whereby women staff wash and sterilize the instruments while male staff help the women patients physically (e.g. getting them into the lithotomy position) when they are most exposed.

\section{Organization and management of camps}

These studies clearly show that the management and organization of sterilization camps is extremely poor. The camps are convened with little planning, and things are often done on an ad hoc basis. There are no well-defined protocols, manuals or guidelines for the staff to follow, no checklist against which to organize the camp, and camp procedures do not appear to be monitored. Because the responsibility for organizing the camps devolves to the various PHCs involved on a rotating basis, overall responsibility for each camp as well as for the specific tasks involved is not clearly defined. No one single individual is in charge of a camp. Senior administrative officials often appear to be more concerned about the camps' ability to meet targets rather than offer quality services.

\section{E. Summary}

From these studies, the variable but often poor quality of services in sterilization camps may be attributed to the intersection of the following factors: inadequate physical infrastructure and logistical support; absence of clear guidelines and protocols setting out standards to be met in services and procedures; an absence of understanding what constitutes quality services; an indifference among many providers to adhering to standards of performance and to the human dimension entailed in providing health services; and finally, the provision of sterilization services largely within the context of meeting targets and thereby achieving volume rather than quality. 


\section{IMPACT OF QUALITY OF CARE ON CONTRACEPTIVE USE}

It has been hypothesized that the quality of care is positively related to a client's level of satisfaction with the services received and to her use of contraception. However, one of the significant findings emerging from the research on clients' perspectives (see Section II) is that Indian women generally express satisfaction with the services received, notwithstanding the fact that they themselves have reported these to be indifferent in the various dimensions of quality, and that this unexpected relationship is a function of the low expectations they hold of services from the government. On the other hand, the studies on the impact of quality of care on contraceptive use presented at this workshop confirm the hypothesized relationship, and demonstrate that high quality care has a direct and positive effect on both contraceptive adoption and continuation. The final section of this report reviews these research findings, drawing on the studies by Koenig, Hossain and Whittaker on Matlab, Bangladesh; Char on urban Bihar, Orissa and Gujarat; Bhat on Karnataka; and Patel on Bharuch district in Gujarat.

\section{A. The Effects of Quality of Care on Contraceptive Adoption}

The study undertaken by Koenig et al focuses on the quality of health and family planning outreach services delivered by female field-workers as measured in a client survey of 7,800 currently married and fecund women in rural Bangladesh, and examines the relationship between these indicators and contraceptive adoption and method continuation. The major findings of their study are as follows:

- Visitation frequency of female field-workers is positively related to contraceptive adoption. Respondents reporting a female fieldworker visitation frequency of at least bimonthly were twice as likely to subsequently adopt a modern contraceptive method as women who had never been visited ( 49 versus 26 percent).

- The duration of an outreach visit is also positively related to subsequent contraceptive adoption. In other words, the more time a fieldworker spends with a client, the greater the likelihood of the latter's adopting a contraceptive method.

- Clients' perceptions of the quality of care provided by field-workers are strongly associated with subsequent contraceptive adoption. Respondents who said they welcomed another visit from the fieldworker were more likely to accept a method than those who viewed another fieldworker visit negatively (55 versus 30 percent). Employing an index of fieldworker quality of care based on respondents' reports of service quality, it was found that high quality service was highly predictive of subsequent contraceptive adoption: women who were classified as having received excellent care were much more likely to adopt contraception than those who were considered to have received poor care (60 versus 25 percent). Controlling for the effects of programmatic variables and client characteristics, multivariate analysis confirms the highly predictive net effect of quality of care on contraceptive adoption. Based on the constructed fieldworker quality of care index, it was found that women who were considered to have received good care were 1.9 times more likely, and excellent care 2.3 times more likely, to subsequently adopt a contraceptive method in comparison to those who 
had received poor care. It is of interest to note that among those who had received substandard care and those who had never been visited by a fieldworker, there was virtually no difference in the rates of subsequent contraceptive adoption. Additionally, the association between perceived quality of care and subsequent contraceptive adoption only exists at the outreach level. There is no association at the clinic level.

- Koenig et al also found that fieldworker quality of care was a much stronger determinant of subsequent contraceptive adoption than contraceptive choice whose relationship to adoption was less clearcut.

\section{B. The Effects of Quality of Care on Contraceptive Continuation}

The individual effects of the different dimensions of quality of care on contraceptive continuation are discussed in the papers by Char, Bhat, and Koenig et al. In these, the researchers identify the specific aspects of care which are significantly associated with the continued use of reversible contraceptive methods. The effects of a program on continuation are discussed in Patel's paper.

Based on interviews with acceptors in urban Bihar, Orissa and Gujarat, Char found that contraceptive continuation is affected by the quality of care in several ways. The contraceptive continuation rate greatly increases if the client is provided with her choice of method. Conversely, a client who has no method in mind when counselled about contraceptive adoption is less likely to continue with a method than one who is using a method of choice. The major reasons given for discontinuation are experience of or anticipation of side effects; however, Char did not correlate discontinuation as a result of side effects with the quality of care variables, follow-up and counselling. In terms of determinants of IUD continuation, Bhat, employing survey data from Karnataka, similarly found that side effects are the major reason for discontinuation in all the use intervals measured. In his analysis, the one quality of care variable which demonstrated a statistically significant effect on continuation was whether the acceptor had received a follow-up visit after insertion, but this was only important in the first three months and only if the visit was made after the first fifteen days following insertion when a woman reportedly becomes aware of side effects.

In assessing whether the impact of quality of care is mediated through side effects, Bhat found that the two variables which were significant were whether the client had received a medical check-up and counselling at the time of acceptance. Koenig et al report that while the association between fieldworker quality of care index and contraceptive continuation is modest for first method use, it is systematic and statistically significant where all method continuation is concerned. After controlling for programmatic and client characteristic variables, clients considered to have received good care are 24 percent, and excellent care 30 percent, more likely to continue using any contraceptive method. Another quality of care variable, the amount of time a fieldworker spends with a client, displays a similar effect on all method continuation. The differential effects on first method continuation and all method continuation may be explained, Koenig et al propose, by the fact that an effective fieldworker will counsel a client who has difficulty with the first method accepted to switch to other more suitable methods rather than discontinuing contraception altogether. 
Patel's paper demonstrates how programmatic interventions may improve contraceptive continuation. She describes how an NGO in rural Gujarat introduced a set of basic and coordinated strategies that educated women on female anatomy and on how the IUD works in relation to their own bodies; this information allayed women's fears and misconceptions about the IUD and raised IUD continuation rates considerably. Although IUD insertions were first performed by this NGO under aseptic conditions, with all the clinical guidelines stringently observed, women were nevertheless extremely apprehensive about this method. A health education program which addressed women's fears and misunderstandings about their bodies was introduced. Employing slides, models and posters, female health workers were able to demonstrate to women the structure of the female anatomy and convince them that since the reproductive system is independent of the gastro-intestinal tract, the IUD will not move from the former to the latter--a commonly held fear.

A simple, low-cost, thermocol model of the uterus, cervix, vagina and fallopian tubes was developed so that all women who sought IUD insertions were first shown the actual procedure on this model. All possible side effects were also discussed with the client at the time of insertion, and clients were instructed to return to the clinic in the event that they experienced any problems. Clinical guidelines for insertions continued to be strictly observed.

Although the proportion of women reporting side effects--leucorrhea, bleeding or pain-did not alter significantly after the interventions were introduced, the rate of IUD removals was significantly lower in the post-intervention group. Among the same group, the rate of IUD continuation was significantly higher for all use intervals when compared to the pre-intervention group: six months after insertion, 72 percent of pre-intervention acceptors and 95 percent of postintervention acceptors still retained their IUDs; after one year the figures were 58 percent and 86 percent respectively; and after two years 42 percent and 77 percent. These findings strongly suggest that interventions such as these which enhance the quality of care within a program, may make a significant contribution to contraceptive continuation.

\section{DISSEMINATION AND UTILIZATION}

The papers presented (see appendix) at the Population Council Workshop on Quality of Services in the Indian Family Welfare Programme are available as working papers, and will be published in book form in 1996. The results have been shared both with the Department of Family Welfare at the union level as well as with State and District authorities in the areas studied. Follow-up is being conducted in demonstration target-free Districts and in the form of district planning exercises in several states.

Considerable work, however, is still required on the methodological aspects of routine data collection on quality, on encouraging supervisors at all levels to use data on quality for program improvement, and on advocacy at the policy level to ensure that standards are explicit, resources are made available and that quality is a valued aspect of family welfare services. 